\title{
Japan Renal Biopsy Registry: the first nationwide, web-based, and prospective registry system of renal biopsies in Japan
}

\author{
Hitoshi Sugiyama $\cdot$ Hitoshi Yokoyama $\cdot$ Hiroshi Sato $\cdot$ Takao Saito $\cdot$ Yukimasa Kohda $\cdot$ Shinichi Nishi $\cdot$ \\ Kazuhiko Tsuruya $\cdot$ Hideyasu Kiyomoto $\cdot$ Hiroyuki Iida $\cdot$ Tamaki Sasaki $\cdot$ Makoto Higuchi $\cdot$ Motoshi Hattori \\ Kazumasa Oka · Shoji Kagami - Michio Nagata - Tetsuya Kawamura • Masataka Honda · Yuichiro Fukasawa • \\ Atsushi Fukatsu $\cdot$ Kunio Morozumi $\cdot$ Norishige Yoshikawa $\cdot$ Yukio Yuzawa $\cdot$ Seiichi Matsuo Yutaka Kiyohara \\ Kensuke Joh-Takashi Taguchi Hirofumi Makino-Committee for Standardization of Renal Pathological \\ Diagnosis and Working Group for Renal Biopsy Database, Japanese Society of Nephrology, Tokyo, Japan
}

Received: 31 May 2010/Accepted: 22 February 2011/Published online: 25 March 2011

(C) Japanese Society of Nephrology 2011

\begin{abstract}
Background The Committee for the Standardization of Renal Pathological Diagnosis and the Working Group for Renal Biopsy Database of the Japanese Society of Nephrology started the first nationwide, web-based, and prospective registry system, the Japan Renal Biopsy Registry (J-RBR), to record the pathological, clinical, and laboratory data of renal biopsies in 2007.

Methods The patient data including age, gender, laboratory data, and clinical and pathological diagnoses were recorded
\end{abstract}

Electronic supplementary material The online version of this article (doi:10.1007/s10157-011-0430-4) contains supplementary material, which is available to authorized users.

\section{H. Sugiyama $\cdot$ H. Makino}

Department of Medicine and Clinical Science, Okayama

University Graduate School of Medicine, Dentistry, and

Pharmaceutical Sciences, Okayama, Japan

\section{H. Yokoyama $(\square)$}

Division of Nephrology, Kanazawa Medical University School of Medicine, 1-1 Daigaku, Uchinada, Ishikawa 920-0293, Japan e-mail: h-yoko@kanazawa-med.ac.jp

\section{H. Sato}

Division of Nephrology, Tohoku University Graduate School of Medicine, Sendai, Japan

\section{T. Saito}

Division of Nephrology and Rheumatology, Department of Internal Medicine, Fukuoka University School of Medicine, Fukuoka, Japan

\section{Y. Kohda}

Department of Nephrology, Faculty of Life Sciences, Kumamoto University, Kumamoto, Japan

\section{S. Nishi}

Blood Purification Center, Niigata University Medical and Dental Hospital, Niigata, Japan on the web page of the J-RBR, which utilizes the system of the Internet Data and Information Center for Medical Research in the University Hospital Medical Information Network. We analyzed the clinical and pathological diagnoses registered on the J-RBR in 2007 and 2008.

Results Data were collected from 818 patients from 18 centers in 2007 and 1582 patients from 23 centers in 2008, including the affiliated hospitals. Renal biopsies were obtained from 726 native kidneys $(88.8 \%)$ and 92 renal grafts $(11.2 \%)$ in 2007 , and 1400 native kidneys $(88.5 \%)$ and 182 renal grafts $(11.5 \%)$ in 2008 . The most common clinical diagnosis was chronic nephritic syndrome (47.4\%), followed by nephrotic syndrome (16.8\%) and renal

\section{K. Tsuruya}

Department of Integrated Therapy for Chronic Kidney Disease, Graduate School of Medical Sciences, Kyushu University,

Fukuoka, Japan

\section{H. Kiyomoto}

Division of Nephrology and Dialysis, Department of Cardiorenal and Cerebrovascular Medicine, Faculty of Medicine, Kagawa University, Kita-gun, Takamatsu, Japan

H. Iida

Department of Internal Medicine, Toyama Prefectural Central Hospital, Toyama, Japan

T. Sasaki

Division of Nephrology and Hypertension, Department of Internal Medicine, Kawasaki Medical School, Kurashiki, Japan

M. Higuchi

Division of Nephrology, Department of Internal Medicine, Shinshu University School of Medicine, Matsumoto, Japan

\section{Hattori}

Department of Pediatric Nephrology, Tokyo Women's Medical University, School of Medicine, Tokyo, Japan 
transplantation (11.2\%) in 2007. A similar frequency of the clinical diagnoses was recognized in 2008. Of the native kidneys, the most frequent pathological diagnosis as classified by pathogenesis was immunoglobulin (Ig) A nephropathy (IgAN) both in 2007 (32.9\%) and 2008 $(30.2 \%)$. Among the primary glomerular diseases (except IgAN), membranous nephropathy (MN) was the most common disease both in 2007 (31.4\%) and $2008(25.7 \%)$. Conclusions In a cross-sectional study, the J-RBR has shown IgAN to be the most common disease in renal biopsies in 2007 and 2008, consistent with previous Japanese studies. MN predominated in the primary glomerular diseases (except for IgAN). The frequency of the disease and the clinical and demographic correlations should be investigated in further analyses by the J-RBR.

Keywords Glomerulonephritis - Tubulointerstitial disorder - Renal vascular disease - Renal grafts · National registry

\section{Introduction}

There has been no national registry of renal biopsies in Japan. The Committee for the Standardization of Renal Pathological Diagnosis and the Working Group for Renal Biopsy Database in the Japanese Society of Nephrology established the first

\section{K. Oka}

Department of Pathology, Osaka Kaisei Hospital, Osaka, Japan

S. Kagami

Department of Pediatrics, Institute of Health Bioscience,

The University of Tokushima Graduate School, Tokushima, Japan

M. Nagata

Molecular Pathology, Biomolecular and Integrated Medical Sciences, Graduate School of Comprehensive Human Sciences, University of Tsukuba, Tsukuba, Japan

T. Kawamura

Division of Kidney and Hypertension, Department of Medicine, Jikei University School of Medicine, Tokyo, Japan

M. Honda

Department of Pediatric Nephrology, Tokyo Metropolitan

Kiyose Children's Hospital, Tokyo, Japan

Y. Fukasawa

Department of Pathology, KKR Sapporo Medical Center,

Sapporo, Japan

\section{A. Fukatsu}

Department of Nephrology, Kyoto University Graduate School of Medicine, Kyoto, Japan

\section{K. Morozumi}

Kidney Center, Japanese Red Cross Nagoya Daini Hospital, Nagoya, Japan nationwide, web-based, and prospective registry system, the Japan Renal Biopsy Registry (J-RBR), to record pathological, clinical, and laboratory data regarding all renal biopsies performed in 2007.

To date, the epidemiological and clinical data of renal diseases are available from nationwide registries of renal biopsies from the United Kingdom [1], Italy [2], Denmark [3], Spain [4], the Czech Republic [5], and Australia [6]. The role of a renal biopsy registry has been recently encouraged [7]. In Japan, several surveys were temporarily conducted for patients with restricted renal diseases, including primary glomerulonephritis [8], idiopathic membranous nephropathy (MN) [9], and immunoglobulin (Ig) A nephropathy (IgAN) [10]. However, there has been no web-based, nationwide, or prospective registry system of overall renal biopsies in Japan. The aim of the current study was to provide data to investigate the epidemiology and frequency of renal diseases with a histological diagnosis for patients registered in 2007 and 2008 on the J-RBR.

\section{Subjects and methods}

Registry system and patients

The researchers on the Committee for the Standardization of Renal Pathological Diagnosis and the Working Group

N. Yoshikawa

Department of Pediatrics, Wakayama Medical University, School of Medicine, Wakayama, Japan

Y. Yuzawa $\cdot$ S. Matsuo

Department of Nephrology, Nagoya University Graduate School of Medicine, Nagoya, Japan

Y. Kiyohara

Department of Environmental Medicine, Graduate School of Medical Sciences, Kyushu University, Fukuoka, Japan

K. Joh

Division of Renal Pathology, Clinical Research Center, ChibaEast National Hospital, Chiba, Japan

T. Taguchi ( $\square)$

Department of Pathology, Nagasaki University Graduate School of Biomedical Sciences, 1-12-4 Sakamoto, Nagasaki 852-8523, Japan

e-mail: taguchi@nagasaki-u.ac.jp

Present Address:

S. Nishi

Division of Nephrology and Kidney Center, Kobe University

Graduate School of Medicine, Kobe, Japan

Present Address:

K. Oka

Department of Pathology, Hyogo Prefectural Nishinomiya

Hospital, Nishinomiya, Japan 
for Renal Biopsy Database in the Japanese Society of Nephrology participated in this study. The report includes the data from patients on the J-RBR, registered prospectively from January to December of 2007 and 2008. Patient data including age, gender, laboratory data, and the clinical and pathological diagnoses were electronically recorded at each institution and registered on the web page of the J-RBR utilizing the system of Internet Data and Information Center for Medical Research (INDICE) in the University Hospital Medical Information Network (UMIN). The ethical committee of the Okayama University Graduate School of Medicine, Dentistry, and Pharmaceutical Sciences comprehensively approved the study, and a local committee of participating centers and their affiliated hospitals individually approved the study. Written informed consent was obtained from the patients at the time of biopsy or before participation in the study. The J-RBR is registered to the Clinical Trial Registry of UMIN (registered number UMIN000000618) and is available in Japanese and English.

Clinical or renal histopathological diagnosis and laboratory data

Three classifications, clinical diagnosis, histological diagnosis by pathogenesis, and histological diagnosis by histopathology, were selected for each case (Supplementary Table) from the J-RBR. The classification of clinical diagnoses was determined as follows: acute nephritic syndrome, rapidly progressive nephritic syndrome, recurrent or persistent hematuria, chronic nephritic syndrome, nephrotic syndrome, renal disorder with metabolic disease, renal disorder with collagen disease or vasculitis, hypertensive nephropathy, inherited renal disease, acute renal failure, drug-induced nephropathy, renal transplantation, and others. The definitions of the former five clinical diagnoses were based on the clinical syndromes and glomerular histopathology in the classification of glomerular diseases [11]. Acute nephritic syndrome was defined as a syndrome characterized by the abrupt onset of hematuria, proteinuria, hypertension, decreased glomerular filtration,

Present Address:

M. Honda

Tokyo Metropolitan Children's Medical Center, Tokyo, Japan

Present Address:

Y. Yuzawa

Department of Nephrology, Fujita Health University, Toyoake, Japan

Present Address:

K. Joh

Division of Pathology, Sendai Shakai Hoken Hospital, Sendai, Japan and edema. Rapidly progressive nephritic syndrome was defined as an abrupt or insidious onset of hematuria, proteinuria, anemia, and rapidly progressing renal failure. Recurrent or persistent hematuria included the insidious or abrupt onset of gross or microscopic hematuria with little or no proteinuria and no evidence of other features of nephritic syndrome. Chronic nephritic syndrome was defined as slowly developing renal failure accompanied by proteinuria, hematuria, with or without hypertension. Nephrotic syndrome was defined as massive proteinuria $>3.5 \mathrm{~g} /$ day and hypoalbuminemia of $<3 \mathrm{~g} / \mathrm{dL}$ of serum albumin with or without edema or hypercholesteremia.

The renal histological diagnosis is classified either according to pathogenesis (A) or by histopathology (B) as follows: (A) primary glomerular disease (except IgAN), IgAN, purpura nephritis, lupus nephritis, myeloperoxidaseantineutrophil cytoplasmic antibody (MPO-ANCA)-positive nephritis, protein 3 (PR3)-ANCA-positive nephritis, anti-glomerular basement membrane antibody nephritis, hypertensive nephrosclerosis, thrombotic microangiopathy, diabetic nephropathy, amyloid nephropathy, Alport syndrome, thin basement membrane disease, infection-related nephropathy, transplanted kidney, and others; (B) minor glomerular abnormalities, focal and segmental glomerulosclerosis (FSGS), MN, mesangial proliferative glomerulonephritis, endocapillary proliferative glomerulonephritis, membranoproliferative glomerulonephritis (MPGN) (type I, III), dense deposit disease, crescentic and necrotizing glomerulonephritis, sclerosing glomerulonephritis, nephrosclerosis, acute interstitial nephritis, chronic interstitial nephritis, acute tubular necrosis, transplanted kidney, and others. IgAN (Berger disease) was separated from primary glomerular diseases on the basis of basic glomerular alterations in the classification of glomerular diseases [11]. Clinical data, including urinalysis, daily proteinuria, serum creatinine concentrations, total protein, albumin, and total cholesterol values were also recorded, but only the frequency of the disease is described here.

\section{Statistics}

Data were expressed as mean $\pm \mathrm{SD}$ as appropriate. Statistical analyses were performed using the JMP software program, version 8 (SAS Institute Inc., Cary, NC, USA).

\section{Results}

Baseline characteristics of registered biopsies

Data were collected from 818 patients from 18 centers in 2007 and 1582 patients from 23 centers in 2008, including the affiliated hospitals. Renal biopsies were obtained from 
Table 1 Number of participating renal centers and registered renal biopsies on the Japan Renal Biopsy Registry (J-RBR) in 2007 and 2008

\begin{tabular}{llll}
\hline Year & 2007 & 2008 & Total \\
\hline Renal centers & 18 & 23 & 23 \\
Total biopsies & 818 & 1582 & 2400 \\
Average age (y) & $44.6 \pm 20.7$ & $44.2 \pm 21.1$ & $44.4 \pm 21.0$ \\
Male & 430 & 851 & 1281 \\
Female & 388 & 731 & 1119 \\
Native kidneys & 726 & 1400 & 2126 \\
Average age (y) & $45.2 \pm 21.4$ & $44.8 \pm 22.0$ & $44.9 \pm 21.5$ \\
Male & 378 & 751 & 1129 \\
Female & 348 & 649 & 997 \\
Renal grafts & 92 & 182 & 274 \\
Average age (y) & $40.5 \pm 13.5$ & $39.4 \pm 16.3$ & $39.8 \pm 15.4$ \\
Male & 52 & 100 & 152 \\
Female & 40 & 82 & 122 \\
\hline
\end{tabular}

726 native kidneys $(88.8 \%)$ and 92 renal grafts $(11.2 \%)$ in 2007 and 1400 native kidneys $(88.5 \%)$ and 182 renal grafts $(11.5 \%)$ in 2008 (Table 1). The average age of the patients was $44.6 \pm 20.7$ years of age in 2007 and $44.2 \pm$ 21.1 years of age in 2008. A higher number of male patients than female patients were registered in both years (male patients $52.6 \%$ in 2007 and $53.8 \%$ in 2008). The distribution of the total number of renal biopsies according to age and gender are presented in Fig. 1, and reveals a different age and gender distribution in native kidneys and renal grafts.

The frequency of clinical diagnoses

The clinical diagnosis and renal histological diagnosis as classified by pathogenesis and by histopathology were determined for each biopsy. A clinical diagnosis of chronic nephritic syndrome was the most frequent, followed by nephrotic syndrome and renal transplantation in 2007, which was similar in 2008 (Table 2). In native kidneys, the majority of the cases corresponded to chronic nephritic syndrome, followed by nephrotic syndrome and recurrent or persistent hematuria or renal disorder with collagen disease or vasculitis in 2007 (Table 2). Similar frequencies of chronic nephritic syndrome, nephrotic syndrome and renal disorder with collagen disease or vasculitis were observed in 2008 (Table 2).

The frequency of pathological diagnoses

Pathological diagnoses were classified by pathogenesis (Table 3) and histopathology (Table 4). In the classification of pathogenesis, IgAN was diagnosed most frequently, followed by primary glomerular disease (except IgAN) and renal grafts both in 2007 and 2008 (Table 3). In the present cohort, except for renal grafts, the frequency of IgAN was $32.9 \%$, followed by primary glomerular disease (except IgAN) (26.3\%) and diabetic nephropathy (5.9\%) in 2007 (Table 3). A slightly lower frequency of IgAN was present $(30.2 \%)$, but similar frequencies of primary glomerular disease (except IgAN) (26.3\%) and diabetic nephropathy $(5.1 \%)$ were observed in 2008 (Table 3).

In the pathological diagnoses as classified by histopathology, mesangial proliferative glomerulonephritis was primarily observed in 2007 and 2008 (Table 4). In the present cohort, except for renal grafts, the frequency of mesangial proliferative glomerulonephritis was the highest followed by $\mathrm{MN}$, minor glomerular abnormalities, nephrosclerosis, and crescentic and necrotizing glomerulonephritis in 2007 (Table 4). In 2008, mesangial proliferative glomerulonephritis was the most frequently diagnosed,
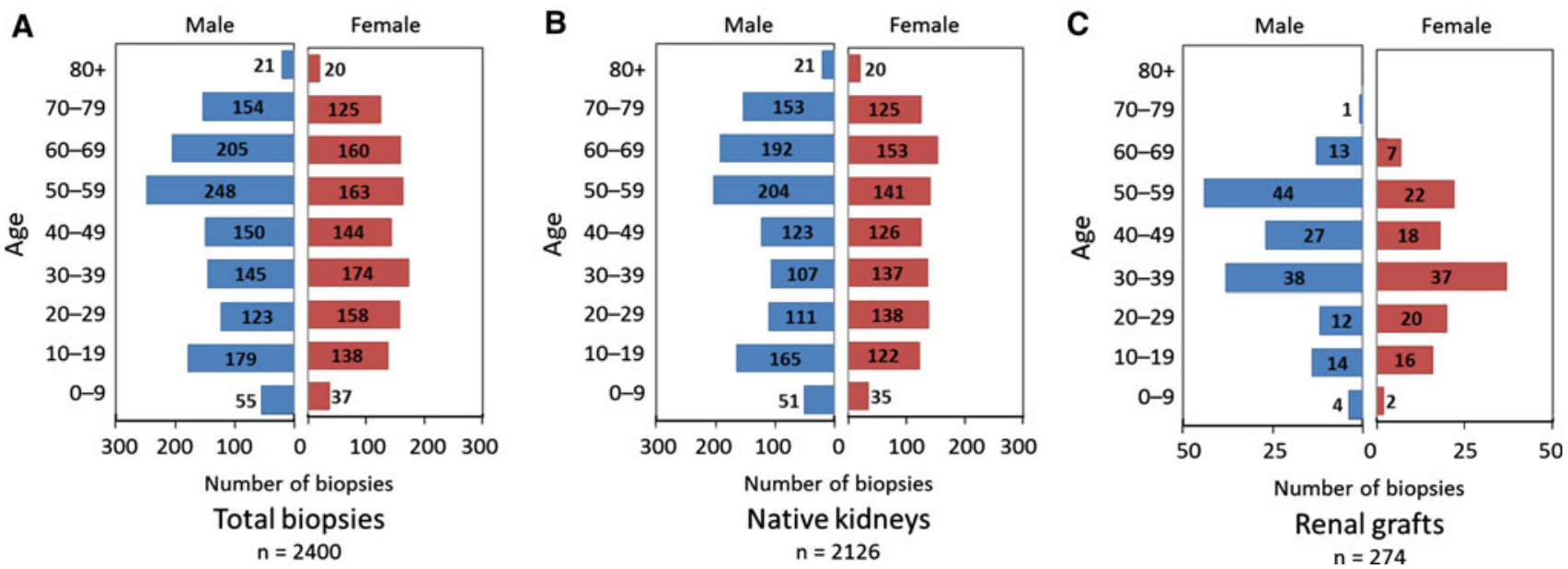

Fig. 1 Distribution of age ranges and gender in total renal biopsies (a), native kidneys (b), and renal grafts (c) in the combined data of 2007 and 2008 
Table 2 Frequency of classification of clinical diagnoses

\begin{tabular}{|c|c|c|c|c|c|c|}
\hline \multirow[t]{2}{*}{ Classification } & \multicolumn{2}{|c|}{2007} & \multicolumn{2}{|l|}{2008} & \multicolumn{2}{|l|}{ Total } \\
\hline & $n$ & $\%$ & $n$ & $\%$ & $n$ & $\%$ \\
\hline Chronic nephritic syndrome & 388 & 47.4 & 768 & 48.5 & 1156 & 48.2 \\
\hline Nephrotic syndrome & 138 & 16.9 & 259 & 16.4 & 397 & 16.5 \\
\hline Renal transplantation & 92 & 11.2 & 182 & 11.5 & 274 & 11.4 \\
\hline Renal disorder with collagen disease or vasculitis & 41 & 5.0 & 87 & 5.5 & 128 & 5.3 \\
\hline Rapidly progressive nephritic syndrome & 33 & 4.0 & 80 & 5.1 & 113 & 4.7 \\
\hline Recurrent or persistent hematuria & 41 & 5.0 & 33 & 2.1 & 74 & 3.1 \\
\hline Renal disorder with metabolic syndrome & 29 & 3.5 & 46 & 2.9 & 75 & 3.1 \\
\hline Hypertensive nephropathy & 14 & 1.7 & 30 & 1.9 & 44 & 1.8 \\
\hline Acute nephritic syndrome & 15 & 1.8 & 20 & 1.3 & 35 & 1.5 \\
\hline Acute renal failure & 7 & 0.9 & 13 & 0.8 & 20 & 0.8 \\
\hline Drug-induced nephropathy & 3 & 0.4 & 11 & 0.7 & 14 & 0.6 \\
\hline Inherited renal disease & 5 & 0.6 & 8 & 0.5 & 13 & 0.5 \\
\hline Others & 12 & 1.6 & 45 & 2.8 & 57 & 2.4 \\
\hline Total & 818 & 100.0 & 1582 & 100.0 & 2400 & 100.0 \\
\hline
\end{tabular}

Table 3 Frequency of pathological diagnoses as classified by pathogenesis

\begin{tabular}{|c|c|c|c|c|c|c|}
\hline \multirow[t]{2}{*}{ Classification } & \multicolumn{2}{|c|}{2007} & \multicolumn{2}{|l|}{2008} & \multicolumn{2}{|l|}{ Total } \\
\hline & $n$ & $\%$ & $n$ & $\%$ & $n$ & $\%$ \\
\hline IgA nephropathy & 239 & 29.2 & 424 & 26.8 & 663 & 27.6 \\
\hline Primary glomerular disease (except IgA nephropathy) & 191 & 23.3 & 369 & 23.3 & 560 & 23.3 \\
\hline Renal graft & 93 & 11.3 & 179 & 11.3 & 272 & 11.3 \\
\hline Diabetic nephropathy & 43 & 5.2 & 71 & 4.5 & 114 & 4.8 \\
\hline Hypertensive nephrosclerosis & 31 & 3.7 & 61 & 3.9 & 92 & 3.8 \\
\hline Lupus nephritis & 29 & 3.5 & 59 & 3.7 & 88 & 3.7 \\
\hline MPO-ANCA-positive nephritis & 25 & 3.0 & 58 & 3.7 & 83 & 3.5 \\
\hline Purpura nephritis & 18 & 2.2 & 39 & 2.5 & 57 & 2.4 \\
\hline Amyloid nephropathy & 12 & 1.4 & 22 & 1.4 & 34 & 1.4 \\
\hline Infection-related nephropathy & 16 & 1.9 & 16 & 1.0 & 32 & 1.3 \\
\hline Thin basement membrane disease & 11 & 1.3 & 5 & 0.3 & 16 & 0.7 \\
\hline Alport syndrome & 1 & 0.1 & 9 & 0.6 & 10 & 0.4 \\
\hline PR3-ANCA-positive nephritis & 1 & 0.1 & 7 & 0.4 & 8 & 0.3 \\
\hline Thrombotic microangiopathy & 3 & 0.3 & 2 & 0.1 & 5 & 0.2 \\
\hline Anti-glomerular basement membrane antibody-type nephritis & 0 & 0.0 & 4 & 0.3 & 4 & 0.2 \\
\hline Others & 105 & 12.8 & 257 & 16.2 & 362 & 15.1 \\
\hline Total & 818 & 100.0 & 1582 & 100.0 & 2400 & 100.0 \\
\hline
\end{tabular}

with minor glomerular abnormalities being the second, and MN being the third (Table 4).

Primary glomerular disease (except IgAN) and nephrotic syndrome

In the cohort of primary glomerular disease as classified by pathogenesis, MN was predominant, followed by mesangial proliferative glomerulonephritis, minor glomerular abnormalities, and FSGS in 2007 (Table 5). In 2008, MN was still the most frequently diagnosed, present at the same frequency as minor glomerular abnormalities (Table 5).

In nephrotic syndrome as classified by clinical diagnosis, primary glomerular disease (except IgAN) was predominant, followed by diabetic nephropathy, amyloid nephropathy, IgAN, and lupus nephritis in 2007 (Table 6). A similar ordering of the disease frequencies was noted in 2008 (Table 6). Among the primary glomerular diseases 
Table 4 Frequency of pathological diagnoses as classified by histopathology

\begin{tabular}{|c|c|c|c|c|c|c|}
\hline \multirow[t]{2}{*}{ Classification } & \multicolumn{2}{|c|}{2007} & \multicolumn{2}{|l|}{2008} & \multicolumn{2}{|l|}{ Total } \\
\hline & $n$ & $\%$ & $n$ & $\%$ & $n$ & $\%$ \\
\hline Mesangial proliferative glomerulonephritis & 326 & 39.8 & 607 & 38.4 & 933 & 38.9 \\
\hline Renal graft & 90 & 11.0 & 171 & 10.8 & 261 & 10.9 \\
\hline Membranous nephropathy & 74 & 9.0 & 128 & 8.1 & 202 & 8.4 \\
\hline Minor glomerular abnormalities & 52 & 6.3 & 143 & 9.0 & 195 & 8.1 \\
\hline Crescentic and necrotizing glomerulonephritis & 32 & 3.9 & 87 & 5.5 & 119 & 5.0 \\
\hline Nephrosclerosis & 38 & 4.6 & 77 & 4.9 & 115 & 4.8 \\
\hline Focal segmental glomerulosclerosis & 32 & 3.9 & 65 & 4.1 & 97 & 4.0 \\
\hline Membranoproliferative glomerulonephritis (type I and III) & 20 & 2.4 & 32 & 2.0 & 52 & 2.2 \\
\hline Chronic interstitial nephritis & 24 & 2.9 & 21 & 1.3 & 45 & 1.9 \\
\hline Endocapillary proliferative glomerulonephritis & 18 & 2.2 & 27 & 1.7 & 45 & 1.9 \\
\hline Sclerosing glomerulonephritis & 10 & 1.2 & 33 & 2.1 & 43 & 1.8 \\
\hline Acute interstitial nephritis & 7 & 0.9 & 18 & 1.1 & 25 & 1.0 \\
\hline Acute tubular necrosis & 5 & 0.6 & 6 & 0.4 & 11 & 0.5 \\
\hline Dense deposit disease & 1 & 0.1 & 5 & 0.3 & 6 & 0.3 \\
\hline Others & 89 & 10.8 & 162 & 10.2 & 251 & 10.5 \\
\hline Total & 818 & 100.0 & 1582 & 100.0 & 2400 & 100.0 \\
\hline
\end{tabular}

Table 5 Frequency of pathological diagnoses as classified by histopathology in primary glomerular disease (except IgA nephropathy)

\begin{tabular}{|c|c|c|c|c|c|c|}
\hline \multirow[t]{2}{*}{ Classification } & \multicolumn{2}{|c|}{2007} & \multicolumn{2}{|c|}{2008} & \multicolumn{2}{|c|}{ Total } \\
\hline & $n$ & $\%$ & $n$ & $\%$ & $n$ & $\%$ \\
\hline Membranous nephropathy & 60 & 31.4 & 95 & 25.7 & 155 & 27.7 \\
\hline Minor glomerular abnormalities & 33 & 17.3 & 95 & 25.7 & 128 & 22.9 \\
\hline Mesangial proliferative glomerulonephritis & 45 & 23.6 & 82 & 22.2 & 127 & 22.7 \\
\hline Focal segmental glomerulosclerosis & 24 & 12.6 & 53 & 14.4 & 77 & 13.8 \\
\hline Membranoproliferative glomerulonephritis (type I and III) & 13 & 6.8 & 19 & 5.1 & 32 & 5.7 \\
\hline Crescentic and necrotizing glomerulonephritis & 5 & 2.6 & 6 & 1.6 & 11 & 2.0 \\
\hline Endocapillary proliferative glomerulonephritis & 1 & 0.5 & 6 & 1.6 & 7 & 1.3 \\
\hline Nephrosclerosis & 2 & 1.0 & 4 & 1.1 & 6 & 1.1 \\
\hline Dense deposit disease & 1 & 0.5 & 3 & 0.8 & 4 & 0.7 \\
\hline Sclerosing glomerulonephritis & 2 & 1.0 & 1 & 0.3 & 3 & 0.5 \\
\hline Others & 5 & 2.6 & 5 & 1.4 & 10 & 1.8 \\
\hline Total & 191 & 100.0 & 369 & 100.0 & 560 & 100.0 \\
\hline
\end{tabular}

(except $\operatorname{Ig} \mathrm{AN}$ ) in nephrotic syndrome, MN was dominant followed by minor glomerular abnormalities, such as minimal change nephrotic syndrome (MCNS), FSGS, and MPGN (type I and III) in 2007 (Table 7). In 2008, the frequency of minor glomerular abnormalities was predominant, followed by MN (Table 7).

Clinical diagnosis of $\mathrm{MN}$, minor glomerular abnormalities, and FSGS

Subanalyses of subjects with a clinical diagnosis of MN, minor glomerular abnormalities, and FSGS were performed since these were the most common forms of primary glomerular diseases (except IgAN) (Tables 8, 9, 10). Nephrotic syndrome was the most common clinical diagnosis in $\mathrm{MN}$ and minor glomerular abnormalities (Tables 8,9), whereas chronic nephritic syndrome was the most common in FSGS (Table 10). In the pathogenesis of minor glomerular abnormalities (total 195 cases), primary glomerular diseases (except IgAN) comprised 65.6\% (128 cases), followed by others $13.8 \%$ (27 cases), IgAN $8.2 \%$ (16 cases) and thin basement membrane disease 5.1\% (10 cases). In the pathogenesis of FSGS (total 97 cases), primary glomerular diseases (except IgAN) comprised 79.4\% 
Table 6 Frequency of pathological diagnoses as classified by pathogenesis in nephrotic syndrome

\begin{tabular}{|c|c|c|c|c|c|c|}
\hline \multirow[t]{2}{*}{ Classification } & \multicolumn{2}{|l|}{2007} & \multicolumn{2}{|l|}{2008} & \multicolumn{2}{|c|}{ Total } \\
\hline & $n$ & $\%$ & $n$ & $\%$ & $n$ & $\%$ \\
\hline Primary glomerular disease (except IgA nephropathy) & 91 & 65.9 & 179 & 69.1 & 270 & 68.0 \\
\hline Diabetic nephropathy & 15 & 10.9 & 15 & 5.8 & 30 & 7.6 \\
\hline Amyloid nephropathy & 9 & 6.5 & 13 & 5.0 & 22 & 5.5 \\
\hline IgA nephropathy & 8 & 5.8 & 9 & 3.5 & 17 & 4.3 \\
\hline Lupus nephritis & 4 & 2.9 & 8 & 3.1 & 12 & 3.0 \\
\hline Purpura nephritis & 1 & 0.7 & 4 & 1.5 & 5 & 1.3 \\
\hline Infection-related nephropathy & 3 & 2.2 & 1 & 0.4 & 4 & 1.0 \\
\hline Thrombotic microangiopathy & 1 & 0.7 & 0 & 0.0 & 1 & 0.3 \\
\hline MPO-ANCA-positive nephritis & 0 & 0.0 & 1 & 0.4 & 1 & 0.3 \\
\hline Hypertensive nephrosclerosis & 0 & 0.0 & 1 & 0.4 & 1 & 0.3 \\
\hline Others & 6 & 4.3 & 28 & 10.8 & 34 & 8.6 \\
\hline Total & 138 & 100.0 & 259 & 100.0 & 397 & 100.0 \\
\hline
\end{tabular}

Table 7 Frequency of pathological diagnoses as classified by histopathology in primary glomerular disease (except IgA nephropathy) in nephrotic syndrome

\begin{tabular}{|c|c|c|c|c|c|c|}
\hline \multirow[t]{2}{*}{ Classification } & \multicolumn{2}{|c|}{2007} & \multicolumn{2}{|l|}{2008} & \multicolumn{2}{|c|}{ Total } \\
\hline & $n$ & $\%$ & $n$ & $\%$ & $n$ & $\%$ \\
\hline Minor glomerular abnormalities & 29 & 31.9 & 79 & 44.1 & 108 & 40.0 \\
\hline Membranous nephropathy & 40 & 44.0 & 56 & 31.3 & 96 & 35.6 \\
\hline Focal segmental glomerulosclerosis & 10 & 11.0 & 25 & 14.0 & 35 & 13.0 \\
\hline Membranoproliferative glomerulonephritis (type I and III) & 7 & 7.7 & 13 & 7.3 & 20 & 7.4 \\
\hline Mesangial proliferative glomerulonephritis & 1 & 1.1 & 4 & 2.2 & 5 & 1.9 \\
\hline Crescentic and necrotizing glomerulonephritis & 2 & 2.2 & 1 & 0.6 & 3 & 1.1 \\
\hline Endocapillary proliferative glomerulonephritis & 1 & 1.1 & 0 & 0.0 & 1 & 0.4 \\
\hline Others & 1 & 1.1 & 1 & 0.6 & 2 & 0.7 \\
\hline Total & 91 & 100.0 & 179 & 100.0 & 270 & 100.0 \\
\hline
\end{tabular}

Table 8 Frequency of clinical diagnoses in membranous nephropathy

\begin{tabular}{|c|c|c|c|c|c|c|}
\hline \multirow[t]{2}{*}{ Classification } & \multicolumn{2}{|c|}{2007} & \multicolumn{2}{|c|}{2008} & \multicolumn{2}{|c|}{ Total } \\
\hline & $n$ & $\%$ & $n$ & $\%$ & $n$ & $\%$ \\
\hline Nephrotic syndrome & 44 & 59.5 & 66 & 51.6 & 110 & 54.5 \\
\hline Chronic nephritic syndrome & 20 & 27.0 & 47 & 36.7 & 67 & 33.2 \\
\hline Renal disorder with collagen disease or vasculitis & 7 & 9.5 & 9 & 7.0 & 16 & 7.9 \\
\hline Renal disorder with metabolic syndrome & 1 & 1.4 & 1 & 0.8 & 2 & 1.0 \\
\hline Recurrent or persistent hematuria & 1 & 1.4 & 0 & 0.0 & 1 & 0.5 \\
\hline Renal transplantation & 0 & 0.0 & 1 & 0.8 & 1 & 0.5 \\
\hline Rapidly progressive nephritic syndrome & 0 & 0.0 & 1 & 0.8 & 1 & 0.5 \\
\hline Acute nephritic syndrome & 0 & 0.0 & 1 & 0.8 & 1 & 0.5 \\
\hline Drug-induced nephropathy & 0 & 0.0 & 1 & 0.8 & 1 & 0.5 \\
\hline Others & 1 & 1.4 & 1 & 0.8 & 2 & 1.0 \\
\hline Total & 74 & 100.0 & 128 & 100.0 & 202 & 100.0 \\
\hline
\end{tabular}


Table 9 Frequency of clinical diagnoses in minor glomerular abnormalities

\begin{tabular}{|c|c|c|c|c|c|c|}
\hline \multirow[t]{2}{*}{ Classification } & \multicolumn{2}{|c|}{2007} & \multicolumn{2}{|c|}{2008} & \multicolumn{2}{|c|}{ Total } \\
\hline & $n$ & $\%$ & $n$ & $\%$ & $n$ & $\%$ \\
\hline Nephrotic syndrome & 29 & 55.8 & 82 & 57.3 & 111 & 56.9 \\
\hline Chronic nephritic syndrome & 9 & 17.3 & 43 & 30.0 & 52 & 26.7 \\
\hline Recurrent or persistent hematuria & 6 & 11.5 & 10 & 7.0 & 16 & 8.2 \\
\hline Renal disorder with collagen disease or vasculitis & 1 & 1.9 & 5 & 3.5 & 6 & 3.1 \\
\hline Rapidly progressive nephritic syndrome & 1 & 1.9 & 0 & 0.0 & 1 & 0.5 \\
\hline Renal disorder with metabolic syndrome & 1 & 1.9 & 0 & 0.0 & 1 & 0.5 \\
\hline Acute nephritic syndrome & 1 & 1.9 & 0 & 0.0 & 1 & 0.5 \\
\hline Drug-induced nephropathy & 1 & 1.9 & 0 & 0.0 & 1 & 0.5 \\
\hline Inherited renal disease & 0 & 0.0 & 1 & 0.7 & 1 & 0.5 \\
\hline Others & 3 & 5.8 & 2 & 1.4 & 5 & 2.6 \\
\hline Total & 52 & 100.0 & 143 & 100.0 & 195 & 100.0 \\
\hline
\end{tabular}

Table 10 Frequency of clinical diagnoses in focal segmental glomerulosclerosis

\begin{tabular}{|c|c|c|c|c|c|c|}
\hline \multirow[t]{2}{*}{ Classification } & \multicolumn{2}{|c|}{2007} & \multicolumn{2}{|c|}{2008} & \multicolumn{2}{|c|}{ Total } \\
\hline & $n$ & $\%$ & $n$ & $\%$ & $n$ & $\%$ \\
\hline Chronic nephritic syndrome & 18 & 56.3 & 32 & 49.2 & 50 & 51.5 \\
\hline Nephrotic syndrome & 10 & 31.3 & 26 & 40.0 & 36 & 37.1 \\
\hline Inherited renal disease & 2 & 6.3 & 0 & 0.0 & 2 & 2.1 \\
\hline Renal disorder with collagen disease or vasculitis & 1 & 3.1 & 1 & 1.5 & 2 & 2.1 \\
\hline Rapidly progressive nephritic syndrome & 1 & 3.1 & 1 & 1.5 & 2 & 2.1 \\
\hline Renal transplantation & 0 & 0.0 & 1 & 1.5 & 1 & 1.0 \\
\hline Recurrent or persistent hematuria & 0 & 0.0 & 1 & 1.5 & 1 & 1.0 \\
\hline Renal disorder with metabolic syndrome & 0 & 0.0 & 1 & 1.5 & 1 & 1.0 \\
\hline Others & 0 & 0.0 & 2 & 3.1 & 2 & 2.1 \\
\hline Total & 32 & 100.0 & 65 & 100.0 & 97 & 100.0 \\
\hline
\end{tabular}

Table 11 Profile of $\operatorname{IgA}$ nephropathy

\begin{tabular}{llll}
\hline IgA nephropathy & 2007 & 2008 & Total \\
\hline Total native kidney biopsies $(n)$ & 239 & 421 & 660 \\
Average age $(\mathrm{y})$ & $36.5 \pm 19.0$ & $36.4 \pm 18.2$ & $36.4 \pm 18.5$ \\
Male $(n)$ & $112(46.9 \%)^{\mathrm{a}}$ & $219(52.0 \%)^{\mathrm{a}}$ & $331(50.2 \%)^{\mathrm{a}}$ \\
Average age $(\mathrm{y})$ & $37.1 \pm 18.9^{\mathrm{b}}$ & $37.2 \pm 19.3^{\mathrm{b}}$ & $37.2 \pm 19.1^{\mathrm{b}}$ \\
Female $(n)$ & $127(53.1 \%)$ & $202(48.0 \%)$ & $329(49.8 \%)$ \\
Average age $(\mathrm{y})$ & $36.1 \pm 19.2$ & $35.4 \pm 17.0$ & $35.7 \pm 17.8$ \\
\hline
\end{tabular}

${ }^{a}$ Ratio indicates percentage of each gender in each biopsy category

b Not significant as compared to another gender

(77 cases), followed by others $11.3 \%$ (11 cases) and hypertensive nephrosclerosis $4.1 \%$ (4 cases).

\section{Subanalysis of IgAN}

The profile, classification of clinical diagnosis, and the pathological diagnosis of $\operatorname{IgAN}$, the most frequent glomerulonephritis on the J-RBR, were further analyzed (Tables 11, 12, 13). The percentage of IgAN detected in total biopsies and native kidneys was 27.5 and $31.0 \%$ in 2007 and 2008, respectively. The average age was the fourth decade in both genders. There was no difference in the proportion based on gender (Table 11). The majority of the clinical and pathological diagnoses were chronic 
Table 12 Frequency of classification of clinical diagnoses in IgA nephropathy

\begin{tabular}{|c|c|c|c|c|c|c|}
\hline \multirow[t]{2}{*}{ Clinical diagnosis } & \multicolumn{2}{|c|}{2007} & \multicolumn{2}{|c|}{2008} & \multicolumn{2}{|c|}{ Total } \\
\hline & $n$ & $\%$ & $n$ & $\%$ & $n$ & $\%$ \\
\hline Chronic nephritic syndrome & 197 & 82.4 & 387 & 91.9 & 584 & 88.5 \\
\hline $\begin{array}{l}\text { Recurrent or persistent } \\
\text { hematuria }\end{array}$ & 23 & 9.6 & 17 & 4.0 & 40 & 6.1 \\
\hline Nephrotic syndrome & 8 & 3.3 & 9 & 2.1 & 17 & 2.6 \\
\hline $\begin{array}{l}\text { Rapidly progressive nephritic } \\
\text { syndrome }\end{array}$ & 8 & 3.3 & 1 & 0.2 & 9 & 1.4 \\
\hline Acute nephritic syndrome & 2 & 0.8 & 4 & 0.9 & 6 & 0.9 \\
\hline Hypertensive nephropathy & 0 & 0.0 & 2 & 0.5 & 2 & 0.3 \\
\hline $\begin{array}{l}\text { Renal disorder with metabolic } \\
\text { disease }\end{array}$ & 1 & 0.4 & 0 & 0.0 & 1 & 0.2 \\
\hline Acute renal failure & 0 & 0.0 & 1 & 0.2 & 1 & 0.2 \\
\hline Total & 239 & 100.0 & 421 & 100.0 & 660 & 100.0 \\
\hline
\end{tabular}

nephritic syndrome (Table 12) and mesangial proliferative glomerulonephritis (Table 13), respectively.

Other diseases

Rare diseases such as Alport syndrome, Fabry disease, lipoprotein glomerulopathy, and dense deposit disease (one case each) were registered in 2007, and one subject was diagnosed with POEMS syndrome in 2008.

\section{Discussion}

The J-RBR obtained data from 818 and 1582 patients with kidney disease and renal transplantation who submitted renal biopsies in 2007 and 2008, respectively. The main objectives of the registry were, based on the histopathological findings, to establish the frequency of glomerulopathies, tubulointerstitial diseases, renal vascular disorders, and renal grafts in renal biopsies in Japan. Data for all patients with histopathological evidence of renal disease at the participating centers were collected on standard forms and registered on the J-RBR program in the UMIN-INDICE. Chronic nephritic syndrome was the most frequent clinical diagnosis in both years of the registry. IgAN was the most frequently diagnosed disease in renal biopsies in 2007 and 2008, consistent with previous reports [8]. In patients with nephrotic syndrome, primary glomerular diseases (except IgAN) were predominant in both years.

Regarding the classification of clinical diagnosis in native kidney biopsies, more than half were diagnosed with chronic nephritic syndrome, which was usually accompanied by urinary abnormalities, as shown in Table 2 . The frequency of clinical diagnosis may reflect the prevalence of renal biopsy in Japan. Indications of renal biopsy in Japan included urinary abnormalities such as mild-tomoderate proteinuria with or without hematuria, massive proteinuria such as nephrotic syndrome, rapidly progressive glomerulonephritis, and renal allografts (a protocol or episode biopsy). Solitary hematuria may be indicated after urological examinations. In Japan, all students in primary and junior high schools routinely undergo an annual urinalysis by the dip-stick test as one of the national health programs. Therafter students in high schools and universities and employees of companies submit to a urinalysis as part of a nationwide screening program. This social system promotes the early referral to nephrologists and may thus influence the frequency of chronic nephritic syndrome according to the clinical diagnoses of the J-RBR.

In the present study, IgAN was the most frequently diagnosed by pathological findings, which is consistent with a previous report [8]. The frequency of IgAN was $32.9 \%$ in 2007 and $30.2 \%$ in 2008 in native kidneys of patients registered on the J-RBR, which was less than that in the previous nationwide survey [8]. IgAN is the most common biopsy-proven renal disease among primary glomerulopathies in Asia as described in reports from

Table 13 Frequency of pathological diagnoses as classified by histopathology

\begin{tabular}{|c|c|c|c|c|c|c|}
\hline \multirow[t]{2}{*}{ Pathological diagnosis by histopathology } & \multicolumn{2}{|c|}{2007} & \multicolumn{2}{|c|}{2008} & \multicolumn{2}{|c|}{ Total } \\
\hline & $n$ & $\%$ & $n$ & $\%$ & $n$ & $\%$ \\
\hline Mesangial proliferative glomerulonephritis & 228 & 95.4 & 398 & 94.5 & 626 & 94.8 \\
\hline Minor glomerular abnormalities & 0 & 0.0 & 16 & 3.8 & 16 & 2.4 \\
\hline Crescentic and necrotizing glomerulonephritis & 2 & 0.8 & 3 & 0.7 & 5 & 0.8 \\
\hline Sclerosing glomerulonephritis & 3 & 1.3 & 0 & 0.0 & 3 & 0.5 \\
\hline Nephrosclerosis & 1 & 0.4 & 1 & 0.2 & 2 & 0.3 \\
\hline Membranous nephropathy & 1 & 0.4 & 1 & 0.2 & 2 & 0.3 \\
\hline Membranoproliferative glomerulonephritis (type I and III) & 1 & 0.4 & 0 & 0.0 & 1 & 0.2 \\
\hline Others & 3 & 1.3 & 2 & 0.5 & 5 & 0.8 \\
\hline Total & 239 & 100.0 & 421 & 100.0 & 660 & 100.0 \\
\hline
\end{tabular}


Korea [12] and China [13]. In the United States, IgAN is the most common primary glomerulopathy in young adult Caucasians and the most common cause of end-stage renal disease, while it was found to be rare in African Americans in whom FSGS remained more common [14]. In Australia, IgAN, FSGS, lupus nephritis, and vasculitis are the most common renal diseases in adults with a male predominance, excepting lupus nephritis [6]. In Europe, IgAN is the most frequent primary glomerulonephritis in several countries $[2,4,5,15]$, while $\mathrm{MN}$ is the most frequent in Macedonia [16], MPGN in Romania [17], and non-IgA mesangial proliferative glomerulonephritis in Serbia [18]. FSGS is the most frequent renal disease in a recent report from Brazil [19]. Because there is a different policy of renal biopsy practice in each country, it may not be easy to compare the different databases across countries. Instead, the changing frequency patterns of renal disease in the same country over a certain time period are useful to treat disease and reduce chronic kidney disease burden [20].

The frequency of nephrotic syndrome was $19.0 \%$ in 2007 and $18.5 \%$ in 2008 for patients registered on the J-RBR. Primary renal diseases were present in approximately two-thirds of all patients with nephrotic syndrome. MN was the most common primary nephrotic syndrome in 2007 (44.0\%) and MCNS was the most common in 2008 $(44.1 \%)$. The reason for this difference may depend on the cohort of registered biopsies in both years, since the number of patients registered was not as large as other registries $[2,4,13,19]$.

For the registry of patients with end-stage renal disease in Japan, there has been a nationwide and yearly statistical survey of chronic dialysis patients since 1968, conducted by the Japanese Society for Dialysis Therapy in Japan [21]. The combined data of the J-RBR with this dialysis registry will allow us to evaluate the long-term outcome of patients with various renal diseases in the near future. Similarly, the combined renal transplant registry data allows the evaluation of patient outcome. A sizeable frequency of renal grafts was registered on the J-RBR. Consequently, the future analysis of renal grafts, including the frequency of the protocol and episode biopsies and the precise histological diagnosis, will be necessary.

There is no overall registry of renal biopsies in Japan at the moment. It is noteworthy that the J-RBR is webbased, and a prospective registry system that can easily increase the number of participating centers and enlarge the number of patients enroled in the future. We cannot conclude that the present sample of patients on the J-RBR in 2007 and in 2008 is actually representative of the nationwide frequency of glomerular, tubulointerstitial, or renal vascular diseases or renal grafts in Japan. However, in the near future, investigation of a larger cohort or a population-based analysis of the rate of each renal disease may reveal the actual frequency of the disease and the distribution of age ranges by utilizing the J-RBR system.

Acknowledgments The authors greatly acknowledge the help and assistance of many colleagues in centers and affiliated hospitals with collecting the data. We also sincerely thank Ms. Mayumi Irie in the UNIN-INDICE for establishing and supporting the registration system of J-RBR. This study was supported by the committee grant from the Japanese Society of Nephrology and by a grant-in-aid from the Research Group on Progressive Renal Disease from the Ministry of Health, Labor and Welfare, Japan.

\section{Appendix}

The following investigators participated in the project for developing the J-RBR:

Hokkaido District

KKR Sapporo Medical Center (Pathology), Akira Suzuki.

\section{Tohoku District}

Tohoku University Hospital and affiliated hospitals (Internal Medicine), Keisuke Nakayama, Takashi Nakamichi.

\section{Kanto District}

Chiba-East National Hospital (Clinical Research Center), Takashi Kenmochi, Hideaki Kurayama, Motonobu Nishimura; The Jikei University Hospital (Internal Medicine); Tokyo Metropolitan Kiyose Children's Hospital (Pediatric Nephrology), Hiroshi Hataya, Kenji Ishikura, Yuko Hamasaki; Tokyo Women's Medical University Hospital (Pediatric Nephrology), Ishizuka Kiyonobu; Tsukuba University Hospital (Pathology and Nephrology), Joichi Usui.

\section{Koushinetsu District}

Niigata University Medical and Dental Hospital (Internal Medicine), Naofumi Imai; Shinshu University Hospital (Internal Medicine), Yuji Kamijo, Wataru Tsukada, Koji Hashimoto.

\section{Hokuriku District}

Kanazawa Medical University Hospital (Internal Medicine), Hiroshi Okuyama, Keiji Fujimoto, Junko Imura; Toyama Prefectural Central Hospital (Internal Medicine), Junya Yamahana, Masahiko Kawabata. 


\section{Tokai District}

Nagoya University Hospital and affiliated hospitals (Internal Medicine), Japanese Red Cross Nagoya Daini Hospital (Kidney Center), Asami Takeda, Keiji Horike, Yasuhiro Otsuka.

\section{Kinki District}

Kyoto University Hospital (Internal Medicine); Osaka Kaisei Hospital (Pathology) and Osaka University Hospital (Internal Medicine), Yoshitaka Isaka, Yasuyuki Nagasawa, Ryohei Yamamoto; Wakayama Medical University Hospital (Pediatrics), Koichi Nakanishi, Yuko Shima.

\section{Chugoku District}

Kawasaki Medical School (Internal Medicine), Naoki Kashihara, Takehiko Tokura; Okayama University Hospital (Internal Medicine), Masaru Kinomura, Hiroshi Morinaga, Tatsuyuki Inoue.

\section{Shikoku District}

Kagawa University Hospital (Internal Medicine and Pathology), Kumiko Moriwaki, Kumiko Kaifu, Yoshio Kushida; Tokushima University Hospital (Pediatrics), Shuji Kondo, Kenichi Suka.

\section{Kyushu District}

Fukuoka University Hospital (Internal Medicine and Pathology), Yoshie Sasatomi, Satoru Ogahara, Satoshi Hisano; Kumamoto University Hospital (Internal Medicine), Kenichiro Kitamura, Yushi Nakayama; Kyushu University Hospital (Internal Medicine), Shunsuke Yamada, Toshiharu Ninomiya; Nagasaki University Hospital (Pathology).

\section{References}

1. Johnston PA, Brown JS, Braumholtz DA, Davison AM. Clinicopathological correlations and long-term follow-up of 253 United Kingdom patients with IgA nephropathy. A report from the MRC Glomerulonephritis Registry. Q J Med. 1992;84:619-27.

2. Schena FP. Survey of the Italian Registry of Renal Biopsies. Frequency of the renal diseases for 7 consecutive years. The Italian Group of Renal Immunopathology. Nephrol Dial Transplant. 1997;12:418-26.

3. Heaf J, Lokkegaard H, Larsen S. The epidemiology and prognosis of glomerulonephritis in Denmark 1985-1997. Nephrol Dial Transplant. 1999;14:1889-97.

4. Rivera F, Lopez-Gomez JM, Perez-Garcia R. Frequency of renal pathology in Spain 1994-1999. Nephrol Dial Transplant. 2002;17:1594-602.
5. Rychlik I, Jancova E, Tesar V, Kolsky A, Lacha J, Stejskal J, Stejskalova A, Dusek J, Herout V. The Czech registry of renal biopsies. Occurrence of renal diseases in the years 1994-2000. Nephrol Dial Transplant. 2004;19:3040-9.

6. Briganti EM, Dowling J, Finlay M, Hill PA, Jones CL, KincaidSmith PS, Sinclair R, McNeil JJ, Atkins RC. The incidence of biopsy-proven glomerulonephritis in Australia. Nephrol Dial Transplant. 2001;16:1364-7.

7. Pesce F, Schena FP. Worldwide distribution of glomerular diseases: the role of renal biopsy registries. Nephrol Dial Transplant. 2010;25:334-6.

8. Nationwide and long-term survey of primary glomerulonephritis in Japan as observed in 1,850 biopsied cases. Research Group on Progressive Chronic Renal Disease. Nephron. 1999;82: 205-13.

9. Shiiki H, Saito T, Nishitani Y, Mitarai T, Yorioka N, Yoshimura A, Yokoyama H, Nishi S, Tomino Y, Kurokawa K, et al. Prognosis and risk factors for idiopathic membranous nephropathy with nephrotic syndrome in Japan. Kidney Int. 2004;65:1400-7.

10. Wakai K, Kawamura T, Endoh M, Kojima M, Tomino Y, Tamakoshi A, Ohno Y, Inaba Y, Sakai H. A scoring system to predict renal outcome in IgA nephropathy: from a nationwide prospective study. Nephrol Dial Transplant. 2006;21:2800-8.

11. Churg J, Bernstein J, Glassock RJ, editors. Renal disease: classification and atlas of glomerular diseases. 2nd ed. New York: IGAKU-SHOIN; 1995. p. 4-20.

12. Chang JH, Kim DK, Kim HW, Park SY, Yoo TH, Kim BS, Kang SW, Choi KH, Han DS, Jeong HJ, et al. Changing prevalence of glomerular diseases in Korean adults: a review of 20 years of experience. Nephrol Dial Transplant. 2009;24:2406-10.

13. Li LS, Liu ZH. Epidemiologic data of renal diseases from a single unit in China: analysis based on 13,519 renal biopsies. Kidney Int. 2004;66:920-3.

14. Nair R, Walker PD. Is IgA nephropathy the commonest primary glomerulopathy among young adults in the USA? Kidney Int. 2006;69:1455-8

15. Simon P, Ramee MP, Boulahrouz R, Stanescu C, Charasse C, Ang KS, Leonetti F, Cam G, Laruelle E, Autuly V, et al. Epidemiologic data of primary glomerular diseases in western France. Kidney Int. 2004;66:905-8.

16. Polenakovic MH, Grcevska L, Dzikova S. The incidence of biopsy-proven primary glomerulonephritis in the Republic of Macedonia-long-term follow-up. Nephrol Dial Transplant. 2003;18(Suppl 5):v26-7.

17. Covic A, Schiller A, Volovat C, Gluhovschi G, Gusbeth-Tatomir P, Petrica L, Caruntu ID, Bozdog G, Velciov S, Trandafirescu V, et al. Epidemiology of renal disease in Romania: a 10 year review of two regional renal biopsy databases. Nephrol Dial Transplant. 2006;21:419-24

18. Naumovic R, Pavlovic S, Stojkovic D, Basta-Jovanovic G, Nesic V. Renal biopsy registry from a single centre in Serbia: 20 years of experience. Nephrol Dial Transplant. 2009;24:877-85.

19. Polito MG, de Moura LA, Kirsztajn GM. An overview on frequency of renal biopsy diagnosis in Brazil: clinical and pathological patterns based on 9,617 native kidney biopsies. Nephrol Dial Transplant. 2010;25:490-6.

20. Imai E, Horio M, Watanabe T, Iseki K, Yamagata K, Hara S, Ura N, Kiyohara Y, Moriyama T, Ando Y, et al. Prevalence of chronic kidney disease in the Japanese general population. Clin Exp Nephrol. 2009;13:621-30.

21. Nakai S, Masakane I, Shigematsu T, Hamano T, Yamagata K, Watanabe Y, Itami N, Ogata S, Kimata N, Shinoda T, et al. An overview of regular dialysis treatment in Japan (as of 31 December 2007). Ther Apher Dial. 2009;13:457-504. 